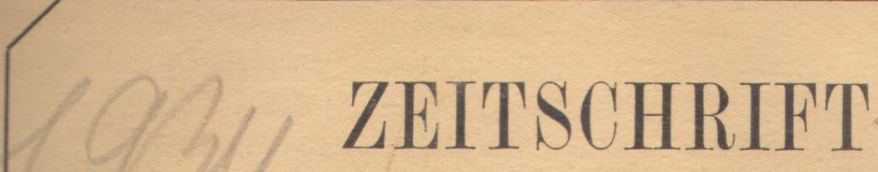 \\ FUR \\ PHYSIKALISCHE CHEMIE \\ BEGRÜNDET VON
}

\author{
WILH. OSTWALD UND J. H. VAN'T HOFF
}

UNTER MITWIRKUNG VON

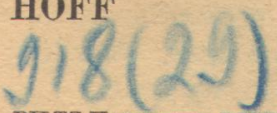

ABEL-WIEN, BAUR-ZÜRICH, BENEDICKS-STOCKHOLM, BENNEWITZ-JENA, BHTZ-HANNOVER, BJERRUM-KOPENHAGEN, BONHÖFFER-BERLIN, BORN-GÖTTINGEN, BRAUNE-HANNOVER, BREDIG-KARLSRUHE, BRONSTED-KOPENHAGEN, CENTNERSZWER-RIGA, CHRISTIANSEN-KOPENHAGEN, COEHNGÖTTINGEN, COHEN-UTREOHT, DEBYE-LEIPZIG, EBERT-WÜRZBURG, EGGERT - LEIPZIG, EUCKENBRESLAU, v. EULER-STOCKHOLM, FAJANS-MÜNOHEN, FOERSTER-DRESDEN, FRANCK-GÖTTINGEN, FREUNDLICH-BERLIN, FÜRTH-PRAG, GERLACH-TUBINGEN, H. GOLDSCHMIDT-OSLO, V. M. GOLDSCHMIDT-OSLO, GRIMM-LUDWIGSHAFEN, HABER-BERLIN, HAHN-BERLIN, v. HALBAN-FRANKFURT A.M., HANTZSCH-LEIPZIG, HENRI-ZÜRICH, HERTZ-BERLIN, HERZFELD-BALTIMORE, v. HEVESY-FREIBURG I. BR., HINSHELWOOD-OXFORD, HORSTMANN - HEIDELBERG, HUND - RosTOCK, HÜTTIG-PRAG, JOFFÉ-LENINGRAD, KALLMANN - BERLIN, KOSSEL-KIEL, KRÜGER-GREIFSWALD, LADENBURGBERLIN, LANDÉ-TUUBINGEN, LE BLANC-LEIPZIG, LE CHATELIER-PARIS, LONDON-BERLIN, LUTHERDRESDEN, MARK-LUDWIGSHAFEN, MECKE-BONN, MEITNER-BERLIN, MEYER-LUDWIGSHAFEN, MITTASCH-OPPAU, MOLES-MADRID, NERNST-BERLIN, PANETH-BERLIN, POLANYI-BERLIN, RIESENFELD-BERLIN, ROTH-BRAUNSCHWEIG, SCHMIDT-MUNSTER, SCHOTTKY-BERLIN, SEMENOFF-LENINGRAD, SIEGBAHN-UPSALA, SMEKAL-HALLE, SVEDBERG-UPSALA, STERN-HAMBURG, TAYLOR-PRINOETON, THIEL-MARBURG, TUBANDT-HALLE, VOLMER-BERLIN, WALDEN-ROSTOCK, v. WARTENBERGDANZIG, WEGSCHEIDER-WIEN, WEIGERT-LEIPZIG, WINTHER-KOPENHAGEN U. ANDEREN FACHGENOSSEN

HERAUSGEGEBEN VON

M. BODENSTEIN - C. DRUCKER • G. JOOS • F. SIMON

ABTEILUNG B:

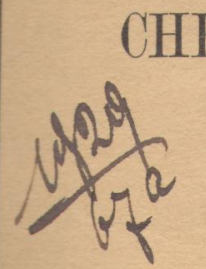

CHEMIE DER ELEMENTARPROZESSE AUFBAU DER MATERIE

SCHRIFTLEITUNG:

M. BODENSTEIN · G. JOOS - F. SIMON

2. BAND, HEFT $1 / 2$

MIT 22 FIGUREN IM TEXT
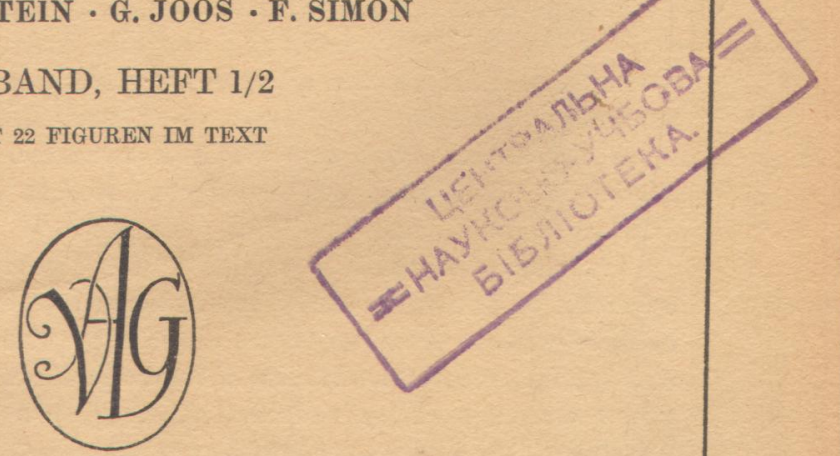

LEIPZIG 1929 • AKADEMISCHE VERLAGSGESELLSCHAFT M. В. H. 


\title{
Die Berechnung der Molekularpolarisation gelöster Stoffe bei unendlicher Verdünnung.
}

\author{
Von \\ Gunnar Hedestrand.
}

(Mit 6 Figuren im Text.)

(Eingegangen am 16. 2. 29.)

Es wird eine Formel hergeleitet, die es ermöglicht, aus den Daten der Dielektrizitätskonstanten und der Dichten von Lösungen die Molekularpolarisation der gelösten Substanz bei unendlicher Verdünnung zu berechnen.

Nach der Clausius-Mosotisischen Theorie der Dielektrika stellt der Ausdruck

$$
P=\frac{\varepsilon-1}{\varepsilon+2} \cdot \frac{M}{d}
$$

ein Mass für den von den Molekülen einer Substanz eingenommenen Raum dar, wenn $\varepsilon$ die Dielektrizitätskonstante (DK), $d$ das spez. Gewicht und $M$ das Molekulargewicht der Substanz bedeuten. Man nennt $P$ die Molekularpolarisation, und nach der Theorie sollte $P$ konstant sein. Das ist aber vielfach nicht der Fall. DeBYE ${ }^{1}$ ) hat in seiner Theorie die Dielektrika durch die Annahme von festen elektrischen Dipolen eine Erklärung der fehlenden Konstanz von $P$ gegeben, und hat auch gezeigt, wie das elektrische Moment dieser Dipole zu berechnen ist. Die Debyesche Theorie bezieht sich in erster Linie auf Gase, weiter aber auch auf verdünnte Lösungen in dipolfreien Lösungsmitteln. Die bereits in grosser Menge vorhandenen Bestimmungen von Dipolmomenten meistens organischer Verbindungen sind in überwiegendem Masse durch die Untersuchung verdünnter Lösungen erhalten.

Für eine binäre Mischung wird die Molekularpolarisation:

$$
P_{1,2}=\frac{\varepsilon-1}{\varepsilon+2} \cdot \frac{f_{1} M_{1}+f_{2} M_{2}}{d}=f_{1} P_{1}+f_{2} P_{2} .
$$

Hier bedeutet $\left.{ }^{2}\right)$ :

$\varepsilon=\mathrm{DK}$ der Lösung,

$d=$ Dichte der Lösung,

$f_{1}, f_{2}=$ Molenbruch der Komponente 1 bzw. 2 (dipolfreies Lōsungsmittel bzw. gelöste Substanz),

1) Debye, Handbuch der Radiologie (MARx) 6, 597. 2) Die hier angewandten Bezeichnungen stimmen hauptsächlich mit denjenigen überein, die von J. W. Williams (Physikal. Ztschr. 29, 174. 1928) benutzt werden. 
$M_{1}, M_{2}=$ Molekulargewicht der Komponente 1 bzw. 2,

$P_{1}, P_{2}=$ Molekularpolarisation der Komponente 1 bzw. 2.

Die Molekularpolarisation der Lösung $P_{1}, 2$ wird somit unter Anwendung eines fiktiven Molekulargewichts $f_{1} \mathrm{M}_{1}+f_{2} M_{2}$ der Lösung berechnet. Wie ersichtlich, ist $P_{1,2}$ eine lineare Funktion von $f_{1}$ und $f_{2}$, wenn $P_{1}$ und $P_{2}$ als konstant angesehen werden können. Für die dipolfreie Substanz dürfte diese Annahme zutreffend sein, wie auch das Experiment zeigt ${ }^{1}$ ). Uns interessiert aber $P_{2}$, die Molekularpolarisation der gelösten Substanz, denn daraus wird das Dipolmoment berechnet. Aus Gleichung (2) bekommt man:

$$
P_{2}=\frac{P_{1,2}-f_{1} P_{1}}{f_{2}}
$$

Die Erfahrung zeigt, dass zwei Fälle vorkommen, wonach wir zwei Klassen von Substanzen unterscheiden:

A. $P_{2}$ ist von der Konzentration unabhängig;

B. $P_{2}$ ist von der Konzentration abhängig.

Die meisten Dipolsubstanzen, gehören zu der Klasse B.

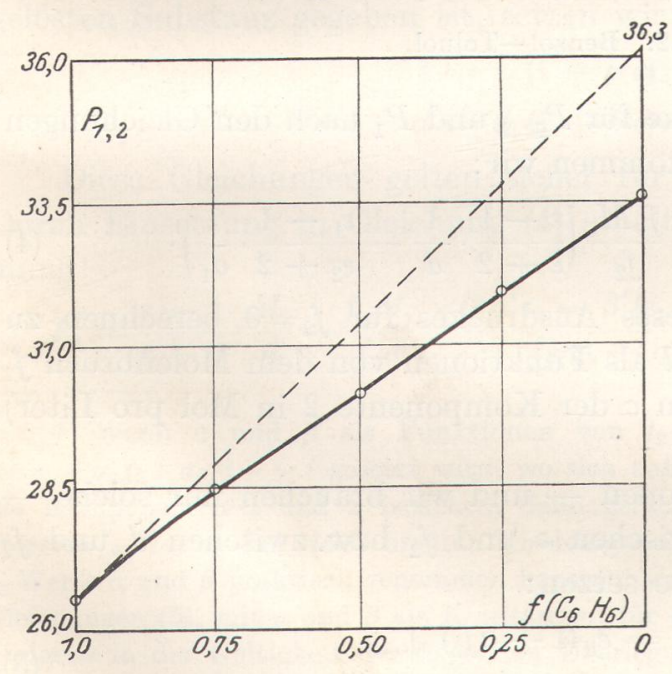

Fig. 1. Benzol-Toluol.

Die Abhängigkeit von $P_{2}$ von der Konzentration führt man auf Assoziation zurück. Zur Berechnung des Dipolmoments wendet man in diesem Falle denjenigen Wert von $P_{2}$ an, den man durch Extrapolation auf unendliche Verdünnung, wo die Assoziation, Null ist, bekommt. Wie diese Extrapolation ausgeführt wird, geht aus Fig. 1 hervor, welche die $P_{1,2}\left(f_{1}\right)$ Kurve des Systems Benzol-Toluol zeigt. Die Tangente der Kurve in dem Punkt $f_{1}=1$ wird ausgezogen, bis sie die Achse $f_{2}=1$ schneidet. Der $P_{1},{ }_{2}$-Wert dieses Schnittpunktes gibt den gesuchten Wert der Molekularpolarisation der zweiten Komponente an.

1) J. RouInski, Physikal. Ztschr. 29, 658. 1928.

Z. physikal. Chem. Abt. B. Bd. 2, Heft 5/6. 
Die Extrapolation ist, wie immer, mit einer gewissen. Unsicherheit behaftet $\left.{ }^{1}\right)$. Eine Kontrolle der Extrapolation kann in vielen Fällen dadurch erzielt werden, dass man die Kurve $P_{2}\left(f_{2}\right)$ zeichnet und aus dieser auf den. Wert für $f_{2}=0$ extrapoliert, wie Fig. 2 zeigt $\left.^{2}\right)$.

Eine nähere Untersuchung von Gleichung (3) zeigt jedoch, dass es möglich ist, unter gewissen Annahmen, die wohl immer zutreffen, die Molekularpolarisation $P_{2}$ bei unendlicher Verdünnung zu berechnen.

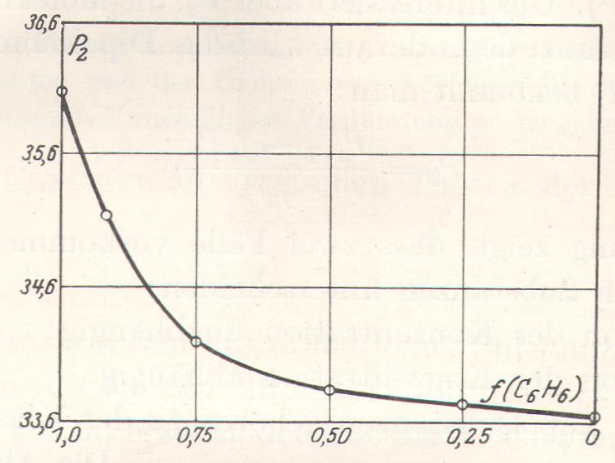

Fig. 2. Benzol-Toluol.

Setzen wir die Ausdrücke für $P_{1},{ }_{2}$ und $P_{1}$ nach den Gleichungeen (2) und (1) in (3) ein, so bekommen wir:

$$
P_{2}=\frac{\varepsilon-1}{\varepsilon+2} \cdot \frac{M_{2}}{d}+\frac{f_{1} M_{1}}{f_{2}}\left(\frac{\varepsilon-1}{\varepsilon+2} \cdot \frac{1}{d}-\frac{\varepsilon_{1}-1}{\varepsilon_{2}+2} \cdot \frac{1}{d_{1}}\right)
$$

Um den Grenzwert dieses Ausdruckes für $f_{2}=0$ berechnen Ix können, müssen wir $\varepsilon$ und $d$ als Funktionen von dem Molenbruch f. (oder von, der Konzentration $c$ der Komponente 2 in Mol pro Liter kennen.

Für kleine Konzentrationen, - und wir brauchen nur solche ist lineare Abhängigkeit zwischen $\varepsilon$ und $f_{2}$ bzw. zwischen $d$ und $f_{\text {. }}$ vorhanden. Wir können also setzen:

$$
\left.\begin{array}{l}
\varepsilon=\varepsilon_{1}\left(1+\alpha f_{2}\right) \\
d=d_{1}\left(1+\beta f_{2}\right)
\end{array}\right\}
$$

wo $\varepsilon_{1}$ bzw. $d_{1} \mathrm{DK}$ bzw. Dichte des reinen Lösungsmittels bedeute-

1) Besonders heikel wird die Sache aber, wenn - wie hier — der erraprilit=Wert durch die Konstruktion der Tangente einer Kurve und weites Awsiture Tangente gefunden wird. ${ }^{2}$ ) Die Fig. 1 und 2 sind der Arbeit rou Ty und Krohмa (J. Amer. Chem. Soc. 49, 1679. 1927) entnommen. 
und $\alpha$ und $\beta$ Konstanten ${ }^{1}$ ) sind, die sowohl positiv als negativ sein können.

Durch Einsetzen von (5) in (4) geht nach einiger Umformung die Gleichung für $P_{2}$ über in:

$$
P_{2}=\frac{\varepsilon-1}{\varepsilon+2} \cdot \frac{M_{2}}{d}+\frac{f_{1} M_{1}}{d} \cdot \frac{3 \alpha \varepsilon_{1}-\beta\left(\varepsilon_{1}-1\right)\left[\varepsilon_{1}\left(1+\alpha f_{2}\right)+2\right]}{\left[\varepsilon_{1}\left(1+\alpha f_{2}\right)+2\right]\left[\varepsilon_{1}+2\right]} .
$$

Setzen wir $\lim _{f_{2} \rightarrow 0} P_{2}=P_{\infty}$ und observieren ferner, dass $\lim _{f_{2} \rightarrow 0} \varepsilon=\varepsilon_{1}$, $\lim d=d_{1}$ und $\lim f_{1}=1$ ist, so bekommen wir nach dem Grenzüber$f_{2} \rightarrow 0$ gange in $(6)$ :

$$
P_{\infty}=\frac{\varepsilon_{1}-1}{\varepsilon_{2}+2} \cdot \frac{M_{2}}{d_{1}}+\frac{M_{1}\left[3 \alpha \varepsilon_{1}-\beta\left(\varepsilon_{1}-1\right)\left(\varepsilon_{1}+2\right)\right]}{d_{1}\left(\varepsilon_{1}+2\right)^{2}}
$$

oder schliesslich:

$$
P_{\infty}=\frac{\varepsilon_{1}-1}{\varepsilon_{1}+2} \cdot \frac{M_{2}-\beta M_{1}}{d_{1}}+\frac{3 M_{1} \alpha \varepsilon_{1}}{d_{1}\left(\varepsilon_{1}+2\right)^{2}} .
$$

Wenn statt der Molenbrüche die Konzentration, $c$ Mol/Liter der gelösten Substanz gegeben ist, setzen wir:

$$
\left.\begin{array}{rl}
\varepsilon & =\varepsilon_{1}\left(1+\alpha^{\prime} c\right) \\
d & =d_{1}\left(1+\beta^{\prime} c\right)
\end{array}\right\}
$$

Diese Gleichungen gelten sicher für kleine Konzentrationen ${ }^{2}$ ). Durch Einsetzung in Gleichung (4) erhält man nach einiger Umformung:

$$
P_{2}=\frac{\varepsilon-1}{\varepsilon+2} \cdot \frac{M_{2}}{d}+\frac{f_{1} M_{1}}{f_{2} d} \cdot \frac{c\left\{3 \alpha^{\prime} \varepsilon_{1}-\beta^{\prime}\left(\varepsilon_{1}-1\right)\left[\varepsilon_{1}\left(1+\alpha^{\prime} c\right)+2\right]\right\}}{\left[\varepsilon_{1}\left(1+\alpha^{\prime} c\right)+2\right]\left[\varepsilon_{1}+2\right]} .
$$

1) Wenn $\alpha$ und $\beta$ als Funktionen von $f_{2}$ angesehen werden, also z. B. $\alpha=\alpha_{0}+\alpha_{1} f_{2}+\alpha_{2} f_{2}^{2}+\cdots$ gesetzt wird, wo sich natürlich $\alpha_{1}$ und $\alpha_{2}$ keineswegs auf die Komponenten 1 und 2 beziehen, dann gelten die Gleichungen (5) auch für grössere $f_{2}$-Werte. Bei vielen binären Mischungen zeigt es sich, dass auch für grosse $f_{2}$-Werte $\alpha$ und $\beta$ praktisch genommen konstant sind. Das Versagen der einfachen Gleichungen (5), mit $\alpha$ und $\beta$ als Konstanten, für grössere Konzentrationen macht übrigens in der Gültigkeit der folgenden Überlegungen gar keine Einschränkung. Wir hätten deshalb ebenso gut

$$
\left.\begin{array}{l}
\varepsilon=\varepsilon_{1}\left(1+\alpha_{0} f_{2}+\alpha_{1} f_{2}^{2}+\cdots\right) \\
d=d_{1}\left(1+\beta_{0} f_{2}+\beta_{1} f_{2}^{2}+\cdots\right)
\end{array}\right\}
$$

ansetzen können.

2) Der Ansatz

$$
\left.\begin{array}{l}
\varepsilon=\varepsilon_{1}\left(1+\alpha_{0}^{\prime} c+\alpha_{1}^{\prime} c^{2}+\cdots\right) \\
d=d_{1}\left(1+\beta_{0}^{\prime} c+\beta_{1}^{\prime} c^{2}+\cdots\right)
\end{array}\right\},
$$

der auch für grössere Konzentrationen gültig ist, ändert in dem Grenzwert $P_{\infty}$ nichts. 
Bezeichnen wir ferner mit $n_{1}$ und $n_{2}$ die Anzahl Mole der Komponenten 1 und 2 im Liter, so ist:

Daraus folgt:

$$
\left.\begin{array}{l}
n_{1}=\frac{1000 d-c M_{2}}{M_{1}} \\
n_{2}=c
\end{array}\right\}
$$

$$
\left.\begin{array}{l}
f_{1}=\frac{n_{1}}{n_{1}+n_{2}}=\frac{1000 d-c M_{2}}{1000 d-c\left(M_{2}-M_{1}\right)} \\
f_{2}=\frac{n_{2}}{n_{1}+n_{2}}=\frac{c M_{1}}{1000 d-c\left(M_{2}-M_{1}\right)}
\end{array}\right\}
$$

und somit:

$$
\frac{f_{1} M_{1}}{f_{2}}=\frac{1000 d-c M_{2}}{c} .
$$

Durch Einsetzen von (11) in (6a) und Grenzübergang bekommen wir schliesslich:

$$
P_{\infty}=\frac{\varepsilon_{1}-1}{\varepsilon_{1}+2} \cdot \frac{M_{2}-1000 \beta^{\prime} d_{1}}{d_{1}}+\frac{3000 \alpha^{\prime} \varepsilon_{1}}{\left(\varepsilon_{1}+2\right)^{2}}
$$

Der Zusammenhang zwischen $\alpha$ und $\alpha^{\prime}$ bzw. $\beta$ und $\beta^{\prime}$ ist durch folgende Gleichungen gegeben:

$$
\alpha^{\prime}=\frac{M_{1}}{1000 d_{1}} \cdot \alpha \text { und } \beta^{\prime}=\frac{M_{1}}{1000 d_{1}} \cdot \beta .
$$

Diese Gleichungen gelten allerdings streng nur für $c=0$, denn sie sind durch Vergleich der Gleichungen (8) und (8a) erhalten ${ }^{1}$ ).

Zur Berechnung der Molekularpolarisation einer gelösten Substanz bei unendlicher Verdünnung werden also aus den DK- und Dichtebestimmungen die Werte der Konstanten $\alpha$ und $\beta$ nach den Gleichungen (5) ermittelt.

Praktischer ist es doch, statt $\alpha$ und $\beta$ selbst, die Werte von $\alpha \varepsilon_{1}$ und $\beta d_{1}$ zu berechnen. Diese gehen, aus der Differenz $\varepsilon-\varepsilon_{1}$ bzw. $d-d_{1}$. nach folgenden Gleichungen hervor ${ }^{2}$ ).

1) Sie gehen auch aus (10), zweiter. Gleichung, für $c=0$ hervor, denn es ist

$$
\lim _{c \rightarrow 0}\left(\frac{f_{2}}{c}\right)=\frac{M_{1}}{1000 d_{1}} .
$$

2) In einigen Methoden zur Bestimmung der DK von Lösungen wird eben die Differenz $\varepsilon-\varepsilon_{1}$ gemessen. Siehe z. B. L. Ebert, R. Eisenschitz u. H. v. Hartz, Z. physikal. Chem. (B) 1, 94. 1928. Man hätte auch den Ansatz $\left.\begin{array}{l}\varepsilon=\varepsilon_{1}+a f_{2} \\ d=d_{1}+b f_{2}\end{array}\right)$ machen können. Dann ist $a=\alpha \varepsilon_{1}$ und $b=\beta d_{1}$. 
Die Berechnung der Molekularpolarisation gelöster Stoffe usw.

$$
\left.\begin{array}{l}
\alpha \varepsilon_{1}=\frac{\varepsilon-\varepsilon_{1}}{f_{2}} \\
\beta d_{1}=\frac{d-d_{1}}{f_{2}}
\end{array}\right\}
$$

bzw. entsprechenden Gleichungen für $\alpha^{\prime} \varepsilon_{1}$ und $\beta^{\prime} d_{1}$. Die Gleichungen (8) und (8a) können nämlich, wie folgt, geschrieben werden:

$$
\begin{aligned}
& P_{\infty}=A \cdot\left(M_{2}-\beta d_{1} \cdot \frac{M_{1}}{d_{1}}\right)+B \cdot \alpha \varepsilon_{1} \\
& P_{\infty}=A \cdot\left(M_{2}-1000 \beta^{\prime} d_{1}\right)+C \cdot \alpha^{\prime} \varepsilon_{1},
\end{aligned}
$$

wo $A, B$ und $C$ für jedes Lösungsmittel bestimmte Konstanten sind:

$$
\left.A=\frac{\varepsilon_{1}-1}{\varepsilon_{1}+2} \cdot \frac{1}{d}^{1}\right) . \quad B=\frac{3 M_{1}}{d_{1}\left(\varepsilon_{1}+2\right)^{2}} . \quad C=\frac{3000}{\left(\varepsilon_{1}+2\right)^{2}} .
$$

Tabelle 1 enthält die Werte dieser Konstanten für die vier gewöhnlichen, dipolfreien, Lösungsmittel Benzol, Hexan, Kohlenstofftetrachlorid und Schwefelkohlenstoff bei $25^{\circ} \mathrm{C}$. Die zur Berechnung benutzten Werte der DK und der Dichte sind den Arbeiten von WпLтAms und Mitarbeitern entnommen ${ }^{2}$ ). Die Werte $\frac{M}{d}$ sind mit Anwendung des aus der Formel abgeleiteten Molekulargewichtes berechnet. Im Falle des Hexans mag das vielleicht nicht ganz richtig sein, denn das mit Hexan bezeichnete Lösungsmittel ist eine bestimmte Fraktion der im Erdöl vorhandenen Kohlenwasserstoffe. Der mögliche Fehler dürfte aber ohne Belang sein (vgl. Tabelle 11).

Tabelle 1.

\begin{tabular}{l|c|c|c|c|c|c}
\hline $\begin{array}{c}\text { Lösungs- } \\
\text { mittel }\end{array}$ & $d_{4}^{25}$ & $\varepsilon$ & $\frac{M}{d}$ & $A$ & $B$ & $C$ \\
\hline$C_{6} H_{6}$ & 0.8731 & $2 \cdot 282$ & 89.40 & 0.3429 & 14.63 & $163 \cdot 6$ \\
$C_{6} H_{14}$ & 0.6776 & 1.904 & 127.1 & 0.3417 & 25.01 & 196.8 \\
$C C_{4}$ & 1.5835 & $2 \cdot 230$ & 97.14 & 0.1836 & 16.29 & 167.7 \\
$C S_{2}$ & 1.2560 & $2 \cdot 633$ & 60.61 & 0.2806 & 8.47 & 139.8 \\
\hline
\end{tabular}

Die folgenden, ziemlich willkürlich gewählten Beispiele mögen als Illustration dienen.

1) $A$ bedeutet also die Grammpolarisation des Lösungsmittels. 2) J. Amer. Chem. Soc. 49, 1676, 2408, 2416. 1927. 50, 94, 362. 1928. 
Tabelle 2. Benzol-Chlorbenzoli).

\begin{tabular}{|c|c|c|c|c|c|}
\hline$f_{2}$ & $d_{4}^{25}$ & $\varepsilon$ & $P_{2}$ & $\beta d_{1}$ & $\alpha \varepsilon_{1}$ \\
\hline \multirow[t]{2}{*}{$\begin{array}{l}0 \\
0.10 \\
0.25 \\
0.50\end{array}$} & $\begin{array}{l}0.8731 \\
0.8993 \\
0.9361 \\
0.9946\end{array}$ & $\begin{array}{l}2.282 \\
2.623 \\
3.131 \\
3.979\end{array}$ & $\begin{array}{l}82.0 \\
77.4 \\
74.5 \\
68.7\end{array}$ & $\begin{array}{c}0 . \overline{262} \\
0.252 \\
0.243\end{array}$ & $\begin{array}{l}3 \cdot \overline{41} \\
3 \cdot 40 \\
3.39\end{array}$ \\
\hline & & & Mit & $0.252 \pm 0.005$ & $3.40 \pm 0.01$ \\
\hline
\end{tabular}

Nach (I) erhält man $P_{\infty}=80 \cdot 6 \pm 0 \cdot 2$, was mit dem extrapolierten Wert 82.0 gut übereinstimmt.

Tabelle 3. Benzol-Benzoesäure ${ }^{2}$ ).

\begin{tabular}{|c|c|c|c|c|}
\hline$f_{2}$ & $\left.d_{4}^{2} 53\right)$ & $\varepsilon$ & $\beta d_{1}$ & $\alpha \varepsilon_{1}$ \\
\hline \multirow[t]{2}{*}{$\begin{array}{l}0.000 \\
0.005 \\
0.010 \\
0.014 \\
0.019 \\
0.024 \\
0.028 \\
0.037 \\
0.046 \\
0.053\end{array}$} & $\begin{array}{l}0.8722 \\
0.8737 \\
0.8754 \\
0.8770 \\
0.8786 \\
0.8802 \\
0.8816 \\
0.8848 \\
0.8878 \\
0.8897\end{array}$ & $\begin{array}{l}2 \cdot 280 \\
2 \cdot 287 \\
2 \cdot 290 \\
2 \cdot 294 \\
2 \cdot 309 \\
2 \cdot 314 \\
2 \cdot 322 \\
2 \cdot 332 \\
2 \cdot 340 \\
2 \cdot 371\end{array}$ & $\begin{array}{l}0.30 \\
0.32 \\
0.34 \\
0.34 \\
0.33 \\
0.34 \\
0.34 \\
0.34 \\
0.33 \\
\end{array}$ & $\begin{array}{l}1.40 \\
1.00 \\
1.00 \\
1.53 \\
1.42 \\
1.50 \\
1.41 \\
1.30 \\
1.72\end{array}$ \\
\hline & & \multicolumn{2}{|c|}{ Mittel: $0.33 \pm 0.01$} & $1.36 \pm 0.08$ \\
\hline
\end{tabular}

$P_{2}($ extrapoliert $\left.)=43 \cdot 5 . \quad P_{\infty}=51 \cdot 6 \pm 1 \cdot 2 .{ }^{4}\right)$

In den Fällen, wo sich $\alpha$ und $\beta$ mit der Konzentration āndern entsteht die Frage, welcher $\alpha$-Wert usw. in die Formeln (I) und (II) einzusetzen ist. Die Antwort ergibt sich aus der Überlegung, dase diese Formeln für $f_{2}=0$ gelten. Wir müssen also die entsprechebden Nullwerte von $\alpha$ und $\beta$ bzw. $\alpha^{\prime}$ und $\beta^{\prime}$ benutzen. Nennen wir dise$\alpha_{0}$ usw. Durch partielle Differentation von (13) ergibt sich:

$$
\alpha_{0} \varepsilon_{1}=\left(\frac{\partial \varepsilon}{\partial f_{2}}\right)_{f_{2}=0} .
$$

Wir zeichnen deshalb die Kurve $\varepsilon$ als Funktion von $f_{2}$ und rît= im Punkte $f_{2}=0$ eine Tangente zu der Kurve (Fig. 3 und 4). Kant=-

1) Winliams und Krohma, J. Amer. Chem. Soc. 49, 1679. 1927. a) Tund Altgeter, J. Amer. Chem. Soc. 49, 2416. 1927. 3) Aus $d^{25}$ 4) Bei der Berechnung wurde das einfache Formelgewicht der Bempeniur gewandt. Nach NeRnst hat aber die Benzoesäure in Benzol ein Molahlungewint:das der doppelten Formel entspricht. Unter dieser Voraussetung elist wis $P_{\infty}=93 \cdot 4$. 
wir den Winkel, welchen diese Tangente mit der $f_{2}$-Achse einschliesst, $\varphi$, dann ist

$$
\alpha_{0} \varepsilon_{1}=\operatorname{tg} \varphi \text {. }
$$

Einfacher und auch sicherer findet man den Wert von $\alpha_{0} \varepsilon_{1}$ durch Extrapolation auf $f_{2}=0$ aus der Kurve $\alpha \varepsilon_{1}$ als Funktion von $f_{2}$ (Fig. 5 und 6$)^{1}$ ). In vielen Fällen ist diese Kurve innerhalb der Versuchsfehler eine gerade Linie und dann ist die Extrapolation. in demselben Masse zuverlässig. Aber auch in den Fällen, wo die Kurve keine Gerade ist, liefert die geradlinige Extrapolation aus den zwei niedrigsten $f_{2}$-Werten, wenn diese z. B. $0 \cdot 1$ und $0 \cdot 25$ sind, den Wert $\alpha_{0} \varepsilon_{1}$ mit ziemlich grosser Sicherheit. Zuweilen ist die Kurve $\alpha^{\prime} \varepsilon_{1}$ als Funktion von $c$ eine gerade Linie mit grösserer Annäherung als die $\alpha \varepsilon_{1}\left(f_{2}\right)$ Kurve, so dass es sich lohnt, diese Möglichkeit zu untersuchen und dadurch eine Kontrolle zu bekommen. Oft ist aber auch das entgegengesetzte

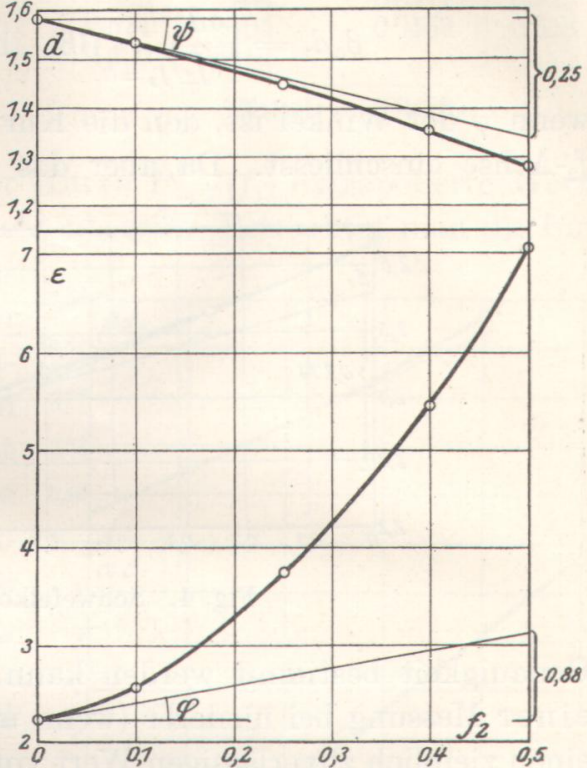

Fig. 3.

Kohlenstofftetrachlorid-Äthylalkohol. der Fall. Wenn mehrere Bestimmungen in dem Bereich $f_{2}<$ etwa $0 \cdot 1$

1) Folgendes Verfahren ist auch anwendbar. Die Neigungen, $\frac{\varepsilon_{n}-\varepsilon_{n-1}}{f_{n}-f_{n-1}}$, der Sehnen werden in Koordinatenpapier als Funktion der mittleren Abszissen, $\frac{f_{n}-f_{n-1}}{2}$ (wenn zur Vermeidung der vielen Indices $f$ statt $f_{2}$ geschrieben wird), eingetragen. Durch die Punktreihe zieht man dann die beste.Gerade, aus deren Schnittpunkt mit der Ordinatenachse der gesuchte Wert $\epsilon_{0} \varepsilon_{1}$ erhalten wird. Dieses Verfahren ist eine der graphischen Methoden zur Bestimmung der Konstanten einer Parabel $\varepsilon=\varepsilon_{1}+a_{0} f+a_{1} f^{2}$. [Ostwald-Luther, Physiko-Chemische Messungen, IV. Aufl., S. 35.] Für Äthylalkohol in Kohlenstofftetrachlorid (Tabelle 4) bekommt man in dieser Weise $a_{0}=1 \cdot 80 \pm 0 \cdot 20$, was mit dem in der Tabelle angegebenen Wert gut übereinstimmt. Die Konstanten der Parabelgleichung $\varepsilon=\varepsilon_{1}+a_{0} f+a_{1} f^{2}$ können übrigens aus den zwei niedrigsten $f$-Werten (z. B. $0 \cdot 1$ und $0 \cdot 25$ ) durch einfache Rechnung bestimmt werden. Entsprechendes gilt für die Parabel $d=d_{1}$ $+b_{0} f+b_{1} f^{2}$. Dann wird jede Extrapolation überflüssig. Für Äthylalkohol 
vorliegen ${ }^{1}$ ), nimmt man einfach den Mittelwert von den daraus berechneten $\alpha \varepsilon_{1}$-Werten, denn die gelegentlichen Schwankungen von $\alpha \varepsilon_{1}$ fallen dann im allgemeinen innerhalb der Versuchsfehler bei den DK-Messungen.

Für $\beta_{0} d_{1}$ gelten entsprechende Gleichungen,

$$
\beta_{0} d_{1}=\left(\frac{\partial d}{\partial f_{2}}\right)_{f_{2}=0}(16) \quad \text { bzw. } \quad \beta_{0} d_{1}=\operatorname{tg} \psi
$$

wenn $\psi$ der Winkel ist, den die Kurve $d$ als Funktion von $f_{2}$ mit der $f_{2}$-Achse einschliesst. Da aber das spez. Gewicht mit viel grösserer

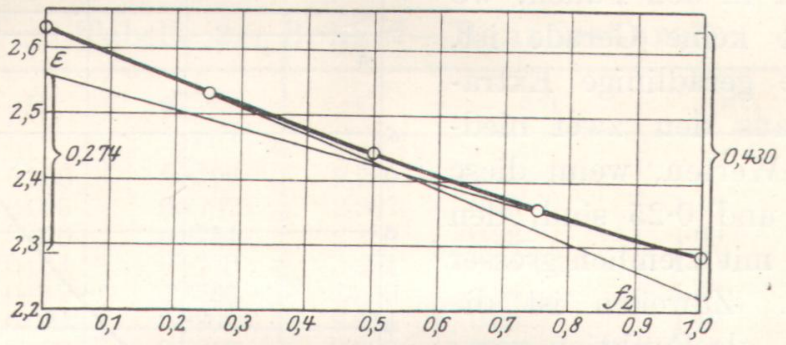

Fig. 4. Schwefelkohlenstoff-Benzol.

Genauigkeit bestimmt werden kann, so dass es möglich ist, aus nur einer Messung bei niederer (wenn nicht allzu niedrig) Konzentration einen ziemlich zuverlässigen Wert von $\beta d_{1}$ zu ermitteln, kann man den so aus Formel $(13,2)$ erhaltenen. Wert benutzen. Besser ist es selbstverständlich, aus mehreren Bestimmungen bei niederer Konzentration einen Mittelwert von $\beta d_{1}$ zu berechnen. Die Anwendung in dem eben besprochenen Fall geht aus den Tabellen 4 und 5 und den dazu gehörigen Fig. 3, 4, 5 und 6 hervor.

Die mit $*$ bezeichneten Werte sind extrapoliert. Es ist somit $\beta_{0} d_{1}=-0 \cdot 492 \pm 0 \cdot 002, \alpha_{0} \varepsilon_{1}=1 \cdot 75 \pm 0 \cdot 10^{2}$ ), woraus $\operatorname{sich} P_{\infty}=45 \cdot 8 \pm 1 \cdot 7$ ergibt. Aus $\beta^{\prime} d_{1}=-0 \cdot 0472 \pm 0 \cdot 0002$ und $\alpha_{0}^{\prime} \varepsilon_{1}=0 \cdot 180 \pm 0 \cdot 015$ erhalt

(Tabelle 4) liefert die Berechnung $a_{0}=\alpha_{0} \varepsilon_{1}=1 \cdot 47$, einen Wert, der mit demjenieren genau übereinstimmt, der durch Extrapolation aus den zwei ersten Punkte= =halten wird (gestrichelte Linie der Fig. 5). Mit $\alpha_{0} \varepsilon_{1}=1 \cdot 47$ und $\beta_{0} d_{1}=-1 /=$ wird $P_{\infty}=41 \cdot 2$ und $\mu=1 \cdot 16 \cdot 10^{-18}$.

1) Siehe Tabelle 3 und ferner in den folgenden Tabellen die Nitrobennils, Nitrotoluole und Dichlorbenzole, Naphthalin, Anthracen, Phenol, Benzoesinte, Zimtsäure und andere. $\left.\quad{ }^{2}\right)$ Die Fehler in $\alpha_{0} \varepsilon_{1}$ usw. sind unter der Anoshme geschätzt worden, dass die DK-Bestimmungen auf $0 \cdot 3 \%$ und die Dichtebestimmungen auf $0.03 \%$ genau sind. 
Die Berechnung der Molekularpolarisation gelöster Stoffe usw.

Tabelle 4. Kohlenstofftetrachlorid-Äthylakohol ${ }^{1}$ ).

(

\begin{tabular}{l|l|l|c|c|c|c|c|c}
\hline$f_{2}$ & \multicolumn{1}{|c|}{$c$} & $d_{4}^{25}$ & $\varepsilon$ & $P_{2}$ & $\beta d_{1}$ & $\alpha \varepsilon_{1}$ & $\beta^{\prime} d_{1}$ & $\alpha^{\prime} \varepsilon_{1}$ \\
\hline 0 & 0 & 1.5835 & 2.230 & 73.5 & $-0.492 *$ & $1.75 *$ & & $0.180^{*}$ \\
0.1 & 1.07 & 1.5325 & 2.560 & 64.9 & 0.510 & 3.30 & -0.0476 & 0.308 \\
0.25 & 2.85 & 1.4491 & 3.74 & 82.8 & 0.538 & $6 \cdot 04$ & 0.0471 & 0.529 \\
0.40 & 4.89 & 1.3540 & 5.45 & 79.7 & 0.574 & 8.05 & 0.0469 & 0.658 \\
0.50 & & 1.2833 & 7.05 & $75 \cdot 2$ & 0.600 & 9.64 & &
\end{tabular}

Mittel: -0.0472

man $P_{\infty}=47 \cdot 3 \pm 2 \cdot 5$. Der aus der Kurve $P_{1,2}\left(f_{1}\right)$ extrapolierte Wert $P_{2}=73 \cdot 5$ stimmt mit diesen, schlecht überein. Betrachtet man die für verschiedene $f_{2}$ berechneten $P_{2}$ Werte, fällt es einem auf, dass der extrapolierte Wert in dem allgemeinen Gang der Kurve $P_{2}$ als Funktion von $f_{2}$ gar nicht passt. Er setzt vielmehr ein Minimum dieser Kurve voraus. Ganz ähnlich sind die Verhältnisse bei Isoamylalkohol. Die oben berechneten Werte $P_{\infty}$ schliessen in sich die Annahme,dass Äthylalkohol in Benzol bei unendlicher Verdünnung ein Molekulargewicht hat, das der einfachen Formel entspricht.

Aus der Tabelle 5 findet man einerseits für Benzol in Schwefelkohlenstoff $\beta_{0} d_{1}=-0 \cdot 640, \alpha_{0} \varepsilon_{1}=$ $-0 \cdot 430$, woraus $P_{\infty}=29 \cdot 2$ berechnet wird. Andererseits erhält man, für Schwefelkohlenstoff in Benzol

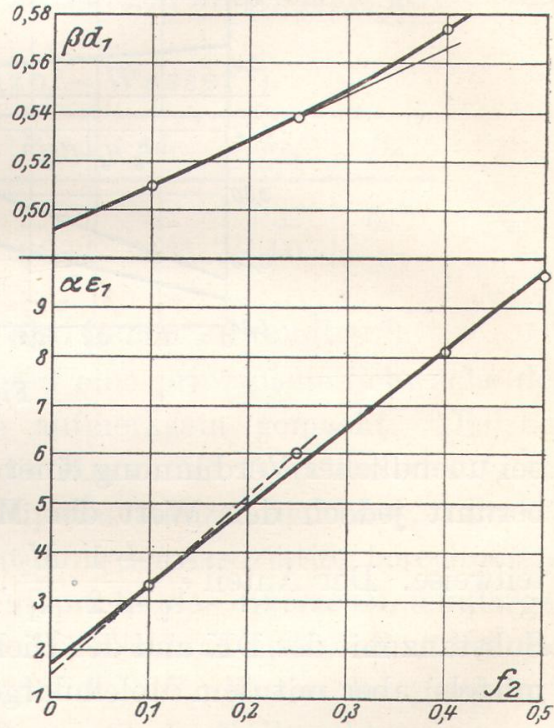

Fig. 5.

Kohlenstofftetrachlorid-Äthylalkohol. $\beta_{0} d_{1}=0 \cdot 268, \alpha_{0} \varepsilon_{1}=0 \cdot 274$ und somit $P_{\infty}=21 \cdot 9$. Hier konnte die Extrapolation aus den $P_{1,2}(f)$ - oder $P_{2}\left(f_{2}\right)$-Kurven mit grosser Sicherheit ausgeführt werden. Die extrapolierten Werte $29 \cdot 0$ bzw. $22 \cdot 2$ stimmen, auch mit den berechneten $29 \cdot 2 \mathrm{bzw} .21 \cdot 9$ ausgezeichnet überein.

In dem zuletzt besprochenen, zweiten Fall bedient sich also auch die hier angegebene Methode zur Ermittlung der Molekularpolarisation

1) KrChMA und Williams, J. Amer. Chem. Soc. 49, 2408. 1927. 
Tabelle 5. Schwefelkohlenstoff-Benzol ${ }^{1}$ ).

\begin{tabular}{l|c|c|c|c|c|r|r}
\hline \multirow{2}{*}{$f_{2}$} & \multirow{2}{*}{$d_{4}^{25}$} & $\varepsilon$ & $P_{2}$ & \multicolumn{2}{|c|}{ Benzol } & \multicolumn{2}{|c}{ Schwefelkohlenstoff } \\
\cline { 5 - 7 } \cline { 5 - 7 } & & & & $\beta d_{1}$ & $\alpha \varepsilon_{1}$ & $\beta d_{1}$ & $\alpha \varepsilon_{1}$ \\
\hline 0 & 1.2561 & 2.633 & 29.0 & $-0.640 *$ & $-0.430 *$ & & \\
0.25 & 1.1167 & 2.531 & 28.4 & 0.558 & 0.408 & 0.326 & 0.328 \\
0.50 & 1.0190 & 2.441 & 27.8 & 0.474 & 0.384 & 0.292 & 0.312 \\
0.75 & 0.9422 & 2.358 & 27.0 & 0.418 & 0.367 & 0.280 & 0.292 \\
1 & 0.8721 & 2.285 & 22.2 & & & $+0.268^{*}$ & $+0.274^{*}$
\end{tabular}

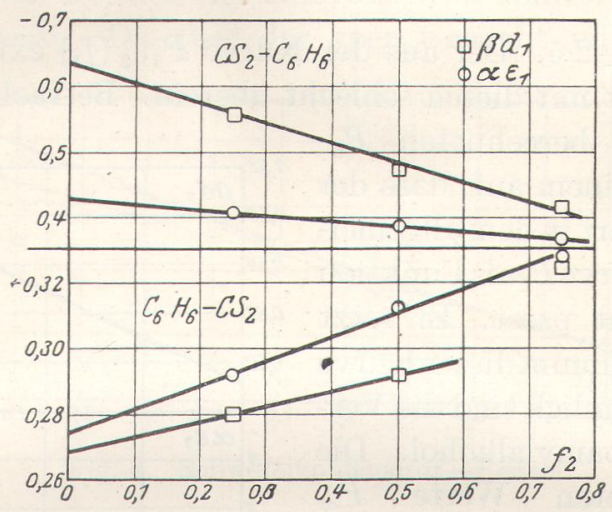

Fig. 6.

bei unendlicher Verdünnung einer graphischen Extrapolation ${ }^{2}$ ). Diese berührt jedoch den Wert der Molekularpolarisation sozusagen nur teilweise. Der Anteil $\frac{\varepsilon_{1}-1}{\varepsilon_{1}+2} \cdot \frac{M_{2}}{d_{1}}$, was die Molekularpolarisation einer Substanz mit der DK und der Dichte der Komponente 1 (des Lösungsmittels) aber mit dem Molekulargewicht der Komponente 2 bedeutet, bleibt davon unberührt. Die Beträge $-\frac{\varepsilon_{1}-1}{\varepsilon_{1}+2} \cdot \frac{\beta M_{1}}{d_{1}}$ und $\frac{3 M_{1} \alpha \varepsilon_{1}}{d_{1}\left(\varepsilon_{1}+2\right)^{2}}$ können indessen kaum als Korrektionsglieder angesehen werden, denn, obwohl ihre Summe bei einigen Substanzen nur wenige Prozente von $P_{\infty}$ ist, machen sie bei anderen fast die ganze Molekularpolarisation aus. Wenn $\alpha$ und $\beta$ gleiche Zeichen haben, wirken sie einander entgegen, wenn aber $\alpha$ und $\beta$ ungleiche Zeichen haben, bewirken sie entweder beide eine Verminderung oder beide eine Vermehrung in $P_{\text {- }}$ -

1) Williams und OGG, J. Amer. Chem. Soc. 50, 94. 1928. In der Abhandlung sind die relativen Dichten $d_{25}^{25}$ mitgeteilt. Diese sind hier auf $d_{4}^{25}$ umgerechnet. 2) Vergleiche Fussnote 1, S. 435 . 
Aus den bekannten Genauigkeiten in den Dichte- und DK-Messungen können die Fehler in $\beta d_{1}$ und $\alpha \varepsilon_{1}$ berechnet werden bzw. in $\beta_{0} d_{1}$ und $\alpha_{0} \varepsilon_{1}$ aus der graphischen Darstellung geschätzt werden. Folglich ist es auch möglich, eine bestimmte Schätzung von dem Fehler in $P_{\infty}$ zu bekommen, eine Schätzung, die bei dem direkten Extrapolationsverfahren sehr willkürlich ist.

Tabelle 6 endlich zeigt den Fall, wo nur eine einzige Bestimmung bei niederer Konzentration vorliegt. Die Extrapolation auf unendliche Verdünnung ist dann aus der $P_{2}\left(f_{2}\right)$-Kurve unmöglich, aus der $P_{1},_{2}\left(f_{1}\right)$ Kurve wohl möglich, aber sehr unsicher. Für die Berechnung nach Formel (I) oder (II) ist es dagegen sogar vorteilhaft, dass die Konzentration gering ist, wenn nur die Messungen hinreichend genau sind.

Tabelle 6. Benzol-Wasser $\left.{ }^{1}\right)$.

\begin{tabular}{l|c|c|c|c|c|c}
\hline \multicolumn{1}{c|}{$f_{2}$} & $d_{4}^{25}$ & $\varepsilon$ & $P_{2}$ & $\beta d_{1}$ & $c \varepsilon_{1}$ & $P_{\infty}$ \\
\hline 0 & 0.8731 & 2.282 & $\overline{-}$ & $-\overline{0}$ & $\overline{4}$ & 63.7 \\
0.0030 & $\mathbf{0 . 8 7 3 2}$ & 2.294 & 64 & 0.033 & -
\end{tabular}

Die Formeln (I) und (II) sind also immer anwendbar ${ }^{2}$ ).

In diesem Zusammenhang sei auf eine prinzipielle Schwäche des direkten Extrapolationsverfahrens aufmerksam gemacht. Die berechneten $P_{1},{ }_{2}$-Werte, aus denen die Kurve als Funktion von $f_{1}$ gezeichnet wird, sind bei assoziierten Dipolsubstanzen nicht die ,,wahren " $P_{1},{ }_{2}$-Werte, d. h. die mit Berücksichtigung der Assoziation berechneten. Denn in Fällen von Assoziation entspricht das fiktive Molekulargewicht $f_{1} M_{1}+f_{2} M_{2}$ der Lösung der Wirklichkeit nicht. Erst bei der Konzentration Null schmiegt sich die $P_{1},_{2}\left(f_{1}\right)$-Kurve der ,wahren" Kurve vollkommen an, so dass die Tangenten im Nullpunkt zusammenfallen. Bei der praktischen Ausführung der Extrapolation dürfte die Sache deshalb keine grosse Rolle spielen. Man hätte aber eigentlich $M_{2}$ als Funktion von $f_{2}$ behandeln sollen, selbstverständlich auch in den hier gemachten Berechnungen von $P_{\infty}$. Da sich aber die Funktion $M_{2}\left(f_{2}\right)$ mit abnehmender Konzentration $M_{2}$ unbegrenzt nähert, wäre dadurch im Grenzwert $P_{\infty}$ nichts geändert worden.

1) J. W. Winliams, Physikal. Ztschr. 29, 204. 1928. $\quad$ 2) Zudem sind sie in der Handhabung sehr bequem, da die Berechnungen von $\beta d_{1}$ und $\alpha \varepsilon_{1}$ aus den entsprechenden Differenzen mit hinreichender Genauigkeit mit dem Rechenstab ausgeführt werden können und die beschwerliche Berechnung von $P_{1},{ }_{2}$ überflüssig wird. 


\section{Berechnungen von Dipolmomenten.}

Die folgenden Tabellen enthalten die berechneten Werte $\beta d_{1}$ und $\alpha \varepsilon_{1}$ bzw. die mit $*$ bezeichneten, extrapolierten Werte $\beta_{0} d_{1}$ und $\alpha_{0} \varepsilon_{1}{ }^{1}$ ) nebst $P_{\infty}$ [nach (I) oder (II) berechnet] für eine grössere Anzahl Substanzen. Zum Vergleich sind die extrapolierten $P_{2}$-Werte mitgenommen. Da der Unterschied zwischen $P_{2}$ (extrapoliert) und $P_{\infty}$ in vielen Fällen ziemlich beträchtlich ist, schien mir eine Neuberechnung der Dipolmomente $\mu$ aus $P_{\infty}$ berechtigt.

Nach Debye kann die Molekularpolarisation in zwei Teile zerlegt werden, eine Verschiebungspolarisation, $P_{E}$, von den Verschie-

Tabelle 7. Lösungsmittel: Benzol.

\begin{tabular}{|c|c|c|c|c|c|c|c|c|}
\hline Molekül & $\begin{array}{c}P_{2} \\
\text { Extrapol. }\end{array}$ & $\beta d_{1}$ & $\alpha \varepsilon_{1}$ & $\begin{array}{l}P_{\infty} \\
\text { ber. }\end{array}$ & Gl. & $P_{E}$ & $P_{0}$ & $\mu \cdot 10^{18}$ \\
\hline Toluol . . . . . . . . . & $36 \cdot 3$ & -0.019 & $0 \cdot 33^{*}$ & 37.0 & I & $30 \cdot 3$ & $6 \cdot 7$ & 0.57 \\
\hline 0 -Xylol $\ldots \ldots \ldots$ & $40 \cdot 8$ & -0.004 & $0 \cdot 20$ & 39.5 & I & & & \\
\hline$o$-Xylol $\ldots \ldots \ldots$ & $40 \cdot 8$ & -0.0004 & $0.017 *$ & 39.3 & II & 35.0 & 4.4 & 0.46 \\
\hline$p$-Xylol $\ldots \ldots \ldots$ & $36 \cdot 7$ & -0.024 & -0.016 & $36 \cdot 9$ & I & 35.5 & 1.4 & 0.26 \\
\hline Nitrobenzol . . . . . . & 348 & 0.392 & 22.5 & 359 & I & $32 \cdot 0$ & 327 & 3.98 \\
\hline$o$-Dinitrobenzol $\ldots \ldots$ & 800 & 0.797 & $52 \cdot 1$ & 795 & I & 35 & 760 & $6 \cdot 05$ \\
\hline$m$-Dinitrobenzol . . . & 338 & 0.781 & 20.8 & 338 & I & 35 & 303 & $3 \cdot 82$ \\
\hline$p$-Dinitrobenzol $\ldots \ldots$ & $37 \cdot 0$ & 0.84 & -0.13 & 30 & I & 34 & 0 & 0.00 \\
\hline 1,3,5-Trinitrobenzol . & $64 \cdot 5$ & 0.83 & 1.42 & $68 \cdot 4$ & I & 40 & 28.4 & 1.17 \\
\hline$o$-Nitrotoluol $\ldots \ldots$ & 331 & 0.387 & $19 \cdot 6$ & 322 & I & 38 & 284 & 3.71 \\
\hline$m$-Nitrotoluol . . . . . & 407 & 0.391 & $27 \cdot 4$ & 436 & I & 38 & 398 & $4 \cdot 38$ \\
\hline$p$-Nitrotoluol . . . . . & 463 & 0.389 & $29 \cdot 3^{*}$ & 464 & I & 38 & 426 & 4.53 \\
\hline Benzoesäure ..... & $43 \cdot 5$ & 0.33 & $1 \cdot 36$ & $51 \cdot 6$ & I & $24 \cdot 6$ & $27 \cdot 0$ & $1 \cdot 14$ \\
\hline$\left(\mathrm{C}_{6} \mathrm{H}_{5} \mathrm{CO}_{2} \mathrm{H}_{2} \quad \ldots \ldots\right.$ & - & 0.33 & $1 \cdot 36$ & $93 \cdot 4$ & I & $49 \cdot 2$ & $44 \cdot 2$ & 1.46 \\
\hline Phenol . . . . . . . & $89 \cdot 0$ & $0 \cdot 20$ & $4 \cdot 2$ & $87 \cdot 6$ & I & 27.7 & $59 \cdot 9$ & 1.70 \\
\hline Chlorbenzol . . . . . . & $82 \cdot 0$ & 0.252 & $3 \cdot 40$ & $80 \cdot 6$ & I & $30 \cdot 2$ & $50 \cdot 4$ & 1.56 \\
\hline Hexan ......... & 30.5 & $-0.270^{*}$ & $0.51 *$ & $30 \cdot 4$ & I & $29 \cdot 6$ & 0.8 & 0.20 \\
\hline Schwefelkohlenstoff . . & $22 \cdot 2$ & $0.268^{*}$ & $0.274^{*}$ & $21 \cdot 9$ & I & $21 \cdot 1$ & 0.8 & 0.20 \\
\hline Chloroform . . . . . & $47 \cdot 5$ & 0.559 & 1.782 & 49.9 & I & 21.3 & 28.6 & 1.17 \\
\hline Chloroform . . . . . & $47 \cdot 5$ & 0.0492 & 0.157 & $49 \cdot 8$ & II & 21.3 & $28 \cdot 6$ & 1.1 \\
\hline Kohlenstofftetrachlorid & $28 \cdot 2$ & 0.769 & -0.066 & 28.2 & I & $28 \cdot 3$ & 0 & 0.00 \\
\hline Äthyläther . . . . . . & 54.5 & $-0.176^{*}$ & $1 \cdot 645^{*}$ & $54 \cdot 8$ & I & $23 \cdot 0$ & 31.8 & 1.22 \\
\hline Wasser ........ & - & 0.033 & $4 \cdot 0$ & 63.7 & I & 4 & 60 & 1.70 \\
\hline Jod . . . . . . . . & $60 \cdot 2$ & $2 \cdot 4$ & $2 \cdot 8$ & 54 & I & 28 & 26 & 1.1 \\
\hline Antimontrijodid .... & - & $\tilde{5}$ & 18 & 282 & I & 52 & 230 & $3 \cdot 3$ \\
\hline Zinnjodid . . . . . & $26 \cdot 7$ & $5 \cdot 1$ & $7 \cdot 3$ & 165 & I & 68 & 97 & $2 \cdot 2$ \\
\hline Silberperchlorat . . . . & 477.0 & 1.7 & 37 & 560 & I & 16 & 544 & 4.9 \\
\hline
\end{tabular}

1) Die Extrapolationswerte sind aus den Kurven $\beta d_{1}$ und $\alpha \varepsilon_{1}$ als Funktionen von $f_{2}$ erhalten worden. 
Tabelle 8. Lösungsmittel: Kohlenstofftetrachlorid.

\begin{tabular}{|c|c|c|c|c|c|c|c|c|}
\hline Molekül & $\begin{array}{c}P_{2} \\
\text { Extrapol. }\end{array}$ & $\beta d_{1}$ & $\alpha \varepsilon_{1}$ & $\begin{array}{l}P_{\infty} \\
\text { ber. }\end{array}$ & Gl. & $P_{E}$ & $P_{0}$ & $\mu \cdot 10^{18}$ \\
\hline Benzol & 26.7 & $-0.66^{*}$ & 0.045 & $26 \cdot 8$ & I & $25 \cdot 8$ & 1.0 & 0.22 \\
\hline Toluol ..... . & 33.7 & -0.7 & 0.161 & $33 \cdot 5$ & I & & $3 \cdot 2$ & 0.39 \\
\hline Chloroform. . & $49 \cdot 8$ & $-0.090^{*}$ & $1.65^{*}$ & 50.4 & I & $21 \cdot 3$ & $29 \cdot 1$ & 1.18 \\
\hline Äthyläther . . . & $56 \cdot 0$ & -0.896 & 1.96 & 61.5 & I & $23 \cdot 0$ & 38 & 1.36 \\
\hline Iethylacetat. . & 78.9 & -0.584 & $3.77 *$ & $85 \cdot 0$ & I & $18 \cdot 0$ & 67 & 1.80 \\
\hline Äthylacetat . . & 87.0 & -0.682 & 3.95 & 92.7 & I & $22 \cdot 2$ & 70.5 & $1 \cdot 84$ \\
\hline Aceton ..... & $170 \cdot 0$ & $-0.614^{*}$ & $10 \cdot 2^{*}$ & 187.8 & I & & $177^{\circ}$ & \\
\hline Aceton ..... & $170 \cdot 0$ & $-0.0596 *$ & 1.000 & 189.1 & II & & & \\
\hline Äthylalkohol . & 73.5 & $-0.492 *$ & $1.75^{*}$ & $45 \cdot 8$ & I & & & \\
\hline Äthylalkohol . & 73.5 & -0.0472 & $0.180^{*}$ & $47 \cdot 3$ & II & & & \\
\hline Isoamylalkohol & $86 \cdot 0$ & $-0.881^{*}$ & $2 \cdot 20^{*}$ & 67.7 & I & 26.81 & 31.2 & 1.23 \\
\hline Isoamylalkohol & - & -0.0857 & $0.205^{*}$ & $66 \cdot 3$ & II & & & \\
\hline
\end{tabular}

Tabelle 9. Lösungsmittel: Schwefelkohlenstoff.

\begin{tabular}{|c|c|c|c|c|c|c|c|c|}
\hline Molekül & $\begin{array}{c}P_{2} \\
\text { Extrapol. }\end{array}$ & $\beta d_{1}$ & $\alpha \varepsilon_{1}$ & $\begin{array}{l}P_{\infty} \\
\text { ber. }\end{array}$ & Gl. & $P_{E}$ & $P_{0}^{\prime}$ & $\mu \cdot 10^{18}$ \\
\hline & 28.5 & & -0.43 & $29 \cdot 2$ & I & $25 \cdot 8$ & $3 \cdot 4$ & 40 \\
\hline robenzol . . & 346 . & & $35.6^{*}$ & 338 & 1 & $32 \cdot 0$ & 306 & $3 \cdot 84$ \\
\hline henol. & $83 \cdot 6$ & -0.2 & $5 \cdot 56$ & $78 \cdot \tilde{0}$ & I & 27.5 & 51.9 & 1.57 \\
\hline Chlorbenzol .. & $82 \cdot 6$ & $-0.290 *$ & $4.92 *$ & $78 \cdot 2$ & I & $31 \cdot 0$ & $47 \cdot 2$ & 1.51 \\
\hline Benzoesäure . . & - & -0.127 & $\left.5 \cdot 4^{2}\right)$ & 82 & I & 24.6 & 57 & 1.7 \\
\hline ure ... & - & $\left.-0 \cdot \tilde{0}^{2}\right)$ & $\left.13 \cdot 3^{2}\right)$ & 162 & I & 41 & 121 & $2 \cdot 4$ \\
\hline ohthal & 54.0 & -0.67 & 1.27 & 58 & I & 4 & 14 & 0.8 \\
\hline Anthracen ... & - & -0.23 & $15 \cdot 4$ & 184 & I & 55 & 129 & 2.5 \\
\hline Hexan ..... & $31 \cdot 6$ & $-1.20 *$ & $-1 \cdot 50 *$ & 31.9 & I & $29 \cdot 6$ & $2 \cdot 3$ & 0.33 \\
\hline Jod . . . . . . & $60 \cdot 0$ & $+3 \cdot 28$ & $5 \cdot 4$ & 61.2 & $\mathrm{I}$ & 28.0 & 33 & 1.3 \\
\hline
\end{tabular}

Tabelle 10. Lösungsmittel: Hexan.

\begin{tabular}{c|c|c|c|c|c|c|c|c}
\hline Molekül & $\begin{array}{c}P_{2} \\
\text { Extrapol. }\end{array}$ & $\beta d_{1}$ & $\alpha \varepsilon_{1}$ & $\begin{array}{c}P_{\infty} \\
\text { ber. }\end{array}$ & Gl. & $P_{E}$ & $P_{0}$ & $\mu \cdot 10^{18}$ \\
\hline Benzol . . . . . . . & 27.8 & 0.156 & 0.303 & 27.5 & I & 25.8 & 1.7 & 0.29 \\
Nitrobenzol . . . . & 346.0 & 0.40 & $13 \cdot 1^{*}$ & 352 & I & 32.0 & 320 & 3.93 \\
Chlorbenzol . . . . & 84.0 & 0.392 & $2.50 *$ & 84.0 & I & 31.0 & 53.0 & 1.60 \\
Benzoesäure . . . . & - & $\left.0.46^{2}\right)$ & $2.92)$ & 94 & I & 24.6 & 69 & 1.8 \\
Naphthalin . . . . . & 55.0 & 0.377 & 1.3 & 60 & I & 44 & 16 & 0.8 \\
Schwefelkohlenstoff . & 29.2 & $0.237 *$ & $0.36^{*}$ & 24.7 & I & 21.1 & 3.6 & 0.42
\end{tabular}

1) J.W. Williams, Physikal. Ztschr. 29, 174. 1928, gibt 16.0 bzw. 13.0 an, was wohl Druckfehler oder Verwechslung zuzuschreiben ist. $\left.\quad{ }^{2}\right)$ Es wurde nur auf die konzentriertesten Lösungen Gewicht gelegt. 
bungen, elektrischer Ladungen im Molekül durch das angelegte, elektrische Feld herrührend, und eine Orientierungspolarisation, $P_{0}$, von dem Richten fertiger Dipole im Felde herrührend. Wir haben somit $P_{\infty}=P_{E}+P_{0}$. Die Grösse $P_{E}$ erhält man aus der Lorenz-Lorentzschen Formel $P_{E}=\frac{n_{0}^{2}-1}{n_{0}^{2}+2} \cdot \frac{M}{d}$, wo $n_{0}$ das auf die Frequenz Null extrapolierte Brechungsindex der Substanz bedeutet. Gewöhnlich begnügt man sich mit dem Wert dieses Ausdruckes für die Natriumlinie ${ }^{1}$ ). Dann ist also $P_{0}=P_{\infty}-P_{E}$ bekannt. Andererseits ist aber nach Debye

$$
P_{0}=\frac{4 \pi N \mu^{2}}{9 k T}
$$

wo $N$ Avogadros Zahl, $k$ Boltzmanns Konstante und $T$ die absolute Temperatur ist. Für $25^{\circ} \mathrm{C}$ gilt dann

$$
\underline{\mu=0.220 \sqrt{P_{0}} \cdot 10^{-18}} .
$$

Die Tabellen 7, 8, 9 und 10 beziehen sich auf die schon zitierten Arbeiten von, W"LLams und Mitarbeitern ${ }^{2}$ ).

Die Tabellen enthalten, wie ersichtlich, einige Substanzen, für die kein $P_{2}$-Wert extrapoliert werden konnte, deren Daten aber eine Berechnung von $P_{\infty}$, wenn auch mit grossem mittleren Fehler, gestatteten. Im Falle des Zinnjodids in Benzol liefert die Berechnung $P_{\infty}=165 \pm 15$ gegenüber $P_{2}$ (extrapoliert) $=26 \cdot 7$ (Rechenfehler?). Sn $J_{4}$ dürfte deshalb polar gebaut sein.

Den Tabellen 12 und 13 liegt die Arbeit von Smyth, Morgan und Boyce $^{3}$ ) zugrunde. Die Atompolarisation, $P_{A}$, ist die aus den Verschiebungen von Atomen und Atomgruppen im Molekül herrührende Polarisation. Die Konstanten A und B haben ein wenig andere Werte als die oben benutzten (Tabelle 11).

Tabelle 14 enthält die entsprechenden Werte einiger Benzolderivate, nach den Messungen von WALDEN und WERnER ${ }^{4}$ ). Gerade diese Arbeit eignet sich für die Berechnung der Molekularpolarisation nach

1) Die Molekularrefraktion einer gelösten Substanz ändert sich gewöhnlich mit der Konzentration. .Der Grenzwert für unendliche Verdünnung kann ans den Messungen von Brechungsindex und Dichte an Lösungen verschiedener Kondectration in analoger Weise berechnet werden, wie hier für die Molekularpolarisation angegeben worden ist. $\quad 2$ ) Zusammenfassende Darstellung von J. W. Wrruturs, Physikal. Ztschr. 29, 174. 1928. 3) Sмyтh, Morgan und Boyce, J. Amer. Chem. Soc. 50, 1536. 1928. 4) P. WALDEN und O. Werner, Z. physikal. Chem. (B) e, 10. 1929. 
Die Berechnung der Molekularpolarisation gelöster Stoffe usw.

Tabelle 11.

\begin{tabular}{c|c|c|r|c|c}
\hline Lösungsmittel & $d_{4}^{25}$ & $\varepsilon$ & $\frac{M}{d}$ & $A$ & $B$ \\
\hline Benzol . . . . . & 0.8727 & 2.273 & 89.4 & 0.3414 & 14.7 \\
Hexan . . . . & 0.6656 & 1.908 & $\mathbf{1 2 9 . 4}$ & 0.3491 & 25.4
\end{tabular}

Tabelle 12. Lösungsmittel: Benzol.

\begin{tabular}{c|c|c|c|c|c|c|c|c}
\hline Molekül & $\begin{array}{c}P_{2} \\
\text { Extrapol. }\end{array}$ & $\beta d_{1}$ & $\kappa \varepsilon_{1}$ & $\begin{array}{c}P_{\infty} \\
\text { ber. }\end{array}$ & Gl. & $P_{E}+P_{A}$ & $P_{0}$ & $\mu \cdot 1018$ \\
\hline Chlorbenzol . . . & 83.6 & 0.282 & 4.03 & 89.1 & $\mathrm{I}$ & $33 \cdot 2$ & 55.9 & 1.64 \\
o-Dichlorbenzol . & 145 & 0.522 & 8.25 & 156 & $\mathrm{I}$ & 40 & 116 & 2.38 \\
m-Dichlorbenzol . & 84.3 & 0.506 & 3.66 & 88.3 & $\mathrm{I}$ & 39.0 & 49.3 & 1.54 \\
p-Dichlorbenzol . & 37.8 & 0.500 & 0.34 & 39.9 & $\mathrm{I}$ & 38.2 & 1.7 & 0.29
\end{tabular}

Tabelle 13. Lösungsmittel: Hexan.

\begin{tabular}{c|c|c|c|c|c|c|c|c}
\hline Molekiil & $\begin{array}{c}P_{2} \\
\text { Extrapol. }\end{array}$ & $\beta d_{1}$ & $\alpha \varepsilon_{1}$ & $\begin{array}{c}P_{\infty} \\
\text { ber. }\end{array}$ & Gl. & $P_{E}+P_{A}$ & $P_{0}$ & $\mu \cdot 1018$ \\
\hline Chlorbenzol .... & - & 0.358 & 2.75 & 92.9 & $\mathrm{I}$ & $33 \cdot 2$ & 59.7 & 1.70 \\
o-Dichlorbenzol . & - & 0.582 & $4.87 *$ & 149 & $\mathrm{I}$ & 40 & 109 & 2.29 \\
m-Dichlorbenzol . & - & $0.635^{*}$ & $4.02 *$ & 125 & $\mathrm{I}$ & 39 & 86 & 2.04
\end{tabular}

Tabelle 14. Lösungsmittel: Benzol.

\begin{tabular}{|c|c|c|c|c|c|c|c|c|}
\hline Molekül & $\begin{array}{c}P_{\infty} \\
\text { Extrapol. }\end{array}$ & $\beta d_{1}$ & $\alpha \varepsilon_{1}$ & $\begin{array}{l}P_{\infty} \\
\text { ber. }\end{array}$ & $\begin{array}{l}\Delta P_{\infty} \\
\text { ber. }\end{array}$ & $P_{E}+P_{A}$ & $P_{0}$ & $\mu \cdot 10^{18}$ \\
\hline Fluorbenzol . . . . & 69 & 0.138 & 2.7 & 67.9 & \pm 3.4 & 28.5 & $39 \cdot 4$ & 1.38 \\
\hline Jodbenzol . . . . . & 73 & 1.18 & $2 \cdot 3$ & 67.2 & 4.5 & 40.1 & $27 \cdot 1$ & 1.14 \\
\hline o-Dibrombenzol. . . & 117 & 1.49 & 5.66 & 118 & 1.9 & $43 \cdot 6$ & 74.4 & 1.89 \\
\hline$m$-Dibrombenzol . . & 93 & 1.3 & 3.9 & 97 & $6 \cdot 6$ & $42 \cdot 2$ & 55 & 1.63 \\
\hline p-Dibrombenzol. . & $40 \cdot 4$ & 1.45 & 0.09 & 37.4 & 1.0 & 40.7 & 0 & 0 \\
\hline o-Bromjodbenzol . . & 112 & 1.92 & 5.20 & 115 & $2 \cdot 3$ & 48.9 & 66.1 & 1.78 \\
\hline$m$-Bromjodbenzol . & 76 & 1.86 & $2 \cdot 6$ & 78 & $6 \cdot 7$ & $48 \cdot 4$ & 30 & 1.20 \\
\hline p-Bromjodbenzol. . & 53 & 1.90 & $1 \cdot 6$ & 62 & $4 \cdot 6$ & 47.9 & 14 & 0.82 \\
\hline o-Chlortoluol .... & $80 \cdot 6$ & 0.244 & 2.9 & 78 & $4 \cdot 4$ & 39.8 & 38 & 1.35 \\
\hline m-Chlortoluol. . . & 95 & 0.240 & $5 \cdot 0$ & 109 & 4.4 & $40 \cdot 6$ & 68 & 1.81 \\
\hline$p$-Chlortoluol .... & 105 & 0.240 & $5 \cdot 20$ & 112 & $2 \cdot 1$ & $41 \cdot 0$ & 71 & 1.85 \\
\hline 0 -Chlornitrobenzol . & 338.5 & $0.88^{*}$ & $21.4^{*}$ & 341 & 8.0 & $36 \cdot 3$ & 305 & 3.84 \\
\hline$m$-Chlornitrobenzol & 250 & 0.65 & $17 \cdot 1$ & 285 & 4.5 & $36 \cdot 4$ & 249 & 3.47 \\
\hline$p$-Chlornitrobenzol. & 154 & $0 \cdot 64$ & 9.3 & 171 & $4 \cdot 4$ & $36 \cdot 4$ & 135 & 2.55 \\
\hline
\end{tabular}

der hier angegebenen Methode ausgezeichnet, denn es sind Messungen an vier bis sieben Lösungen in dem Konzentrationsbereich entsprechend $f_{2}=0-0.15$ gemacht worden. Die Konstanten der Formel (I) haben 
444 Gunnar Hedestrand, Die Berechnung der Molekularpolarisation usw.

hier folgende Werte: $A=0 \cdot 3374, B=14 \cdot 69$. Die Übereinstimmung zwischen $P_{\infty}$ (ber.) und $P_{\infty}$ (extrapoliert) ist meistens gut. Der aus den mittleren Fehlern von $\beta d_{1}$ und $\alpha \varepsilon_{1}$ berechnete Fehler in $P_{\infty}$ (ber.), $\Delta P_{\infty}$, zeigt, dass der angegebene Fehler in $P_{\infty}$ (extrapoliert), $\pm 2 \mathrm{~cm}^{3}$, ein wenig zu optimistisch geschätzt worden ist.

Schliesslich seien noch die Berechnungen für Glykollösungen in Wasser angeführt (Tabelle 15) ${ }^{1}$ ).

Tabelle 15. Glykokoll-Wasser.

\begin{tabular}{|c|c|c|c|c|c|}
\hline$c$ & $d_{4}^{18}$ & $\beta^{\prime} d_{1}$ & $\varepsilon$ & $c^{\prime} \varepsilon_{1}$ & $P_{2}$ \\
\hline $\begin{array}{l}0 \\
0.1 \\
0.25 \\
0.5 \\
1\end{array}$ & $\begin{array}{l}1.0000 \\
1.0018 \\
1.0050 \\
1.0145 \\
1.0300\end{array}$ & $\begin{array}{l}0.015^{*} \\
0.018 \\
0.020 \\
0.02 .9 \\
0.030\end{array}$ & $\begin{array}{r}81.0 \\
83.1 \\
86.7 \\
92.3 \\
104.1\end{array}$ & $\begin{array}{l}2 \overline{21.0} \\
22.8 \\
22.6 \\
23.1\end{array}$ & $\begin{array}{l}67-7 \\
641 \\
55-7 \\
53-1 \\
51.3\end{array}$ \\
\hline
\end{tabular}

Da aber hier das Lösungsmittel selbst eine Dipolsubstanz ist und, deshalb die Deвyesche Theorie nicht anwendbar ist, kann aus der berechneten, Molekularpolarisation, $P_{\infty}=67 \cdot 7$, das Dipolmoment des gelösten Moleküls nicht berechnet werden. Im Zusammenhang mit ferneren Messungen wird später auf diese Frage eingegangen werden.

\section{Zusammenfassung.}

Durch geeignete Ansätze, welche die DK und die Dichte einer Lösung als Funktionen von, der Konzentration darstellen, wurde der Grenzwert der Molekularpolarisation der gelösten Substanz bei unendlicher Verdünnung berechnet.

Die Anwendung der hergeleiteten Formeln wurde an verschiedenen Beispielen gezeigt, und die Resultate der Berechnungen von Molekularpolarisation und Dipolmoment wurden für eine Anzahl Substanzen in Tabellen zusammengestellt.

1) G. Hedestrand, Z. physikal. Chem. 135, 36. 1928.

Stockholm, Institut f. allgem. u. organ. Chemie d. Universität. Februar 1929. 